\title{
Utilizing a Qualitative and Quantitative Approach to Examine Substance Use and Parental Communication among African American Youth
}

\author{
Kimberly Boyd*, Patrice Perkins, Byron Greenberg, Leah Stevens \\ Department of Psychology, Virginia State University, Petersburg, USA \\ Email: ${ }^{*}$ kboyd@vsu.edu
}

Received 12 August 2014; revised 5 September 2014; accepted 29 September 2014

Copyright (C) 2014 by authors and Scientific Research Publishing Inc.

This work is licensed under the Creative Commons Attribution International License (CC BY). http://creativecommons.org/licenses/by/4.0/

\section{(c) (7) Open Access}

\begin{abstract}
Objective: The present study examined the communication patterns between children and parents regarding substance use and abuse issues. Method: The participants for the study were 111 youth and young adults $(59.8 \%$ female/40.2\% male). The youth were between the ages of $13-18$. Results: Findings from the study supported the hypothesis. Results demonstrated that higher family cohesion suggested lower drug use. However, findings also identified a potential gap in the communication process among parents and youth. The majority of the youth from the qualitative analysis who admitted to using drugs stated that their parents usually say, "Do not use drugs." However, their parents "have never provided open discussions about drugs." Conclusion: This project expands our knowledge on parental communication and drug use among youth. A continuation of this research would assist with providing a deeper understanding of the parent-child relationship and drug use and abuse among African American youth.
\end{abstract}

\section{Keywords}

Substance Use, African American Youth, Parent-Child Communication

\section{Introduction}

In 2001, the Substance Abuse and Mental Health Services Administration (SAMHSA) estimated that 6.1 million

\footnotetext{
${ }^{*}$ Corresponding author.
}

How to cite this paper: Boyd, K., Perkins, P., Greenberg, B., \& Stevens, L. (2014). Utilizing a Qualitative and Quantitative Approach to Examine Substance Use and Parental Communication among African American Youth. Psychology, 5, 17431749. http://dx.doi.org/10.4236/psych.2014.515181 
youth aged 12 - 17 had problems with drugs that warranted treatment. SAMHSA also predicted that substance use disorders will rise among this population. Between 2003 and 2005, substance use disorders increased by 1 million people (SAMHSA, 2003). Dawson, Grant, Stinson, \& Chou (2004) offered that approximately $18 \%$ of college students (ages 18 - 24) met the Diagnostic and Statistical Manual of Mental Disorders (DSM-IV) (American Psychiatric Association, 2000) criteria for alcohol abuse or dependence within the previous year. This abuse and dependence does not occur overnight and is likely evolving during late childhood and early adolescence.

The National Survey on Drug Use and Health echoes these startling rates among youth with major mental health concerns. In 2011, 36\% of youth aged 12 - 17 diagnosed with a major depressive episode used illicit drugs in the past year (SAMHSA, 2012). This problem crosses all ethnic and racial groups, but appears to affect some groups more acutely. Pacek, Malcolm, \& Martins (2012) suggested that while alcohol use disorders were comparable between Caucasians, Hispanics, and African Americans in their sample, marijuana use disorders were more common among African Americans. In addition, co-occurring alcohol and marijuana use disorders were more prevalent in African Americans compared to Caucasians and Hispanics (Pacek et al., 2012). The age adjusted death rate due to substance use disorders for African Americans is 31\% greater than for the general population (SAMHSA, 2003).

Parent-child communication around substance use has been a subject of discussion for many years (Demant \& Ravn, 2013; Elkington, Bauermeister, \& Zimmerman, 2011; Luk, Farhat, Iannotti, \& Simons-Morton, 2010; Miller-Day \& Kam, 2010). Research has suggested that parental substance use and the availability of alcohol and other drugs such as marijuana influence the onset of drug use among teens (Bauermeister et al., 2012; Haas, Smith, Kagan, \& Jacob, 2012; Stanton et al., 2004). A considerable number of studies suggest that parental attitudes about drug use play a major role in the parent-child arena. Luk et al. (2010) suggested that parent-child communication could be a modifying protective factor against substance use practices. This study suggested that the frequency and quality of the parent-child communication was associated with tobacco use among mothers and sons. Thus, stronger mother-son communication resulted in lower tobacco use.

Family process theory suggests that open communication patterns encourage children and youth to internalize the values and norms communicated within the parents' messages (Whitaker \& Miller, 2000). Wills, Gibbons, Gerrard, Murry, \& Brody (2003) offered a theoretical model for path analysis that included a measure of parent-adolescent communication as an influence on drug use. Their results suggest that this communication process had its greatest effect on drug use outcomes, further supporting the present research and offering a potential area of intervention.

Some studies have suggested that communication by itself may increase drinking behavior among adolescent college students, but specific types of communication may actually lower risk of use or abuse (Turrisi, Mastroleo, Mallett, Larimer, \& Kilmer, 2007). The question of interest in this study is: Can specific elements of the parent-child communication and drug use discussion be identified to assist with the development of effective interventions for families? One thing seems clear from the current literature; it is not simply a matter of a general discussion about drugs that influences the drug use frequency among youth. There are many factors associated with the parent-child relationship and communication patterns that must be examined. Demant \& Ravn (2013) identified family cohesion and parental trust as two factors that play a role in parent-child communication and drug use. This study will seek to identify some of the particular characteristics of the communication and then quantify some of these variables for analytical purposes.

It is clear that a relationship between parental communication and drug use exists; yet, studying this relationship quantitatively may not address the entire issue. To this end, the present study examined parent-child communication and cohesion both qualitatively and quantitatively to assist with providing a bigger picture and exploring the issue in-depth. The following hypotheses will be examined: 1) Parent-child communication will play a major role in the onset of substance use among youth. Specifically, higher family cohesion, family communication, and stronger parental relationships will result in lower drug use and higher family cohesion, family communication, and stronger parental relationships will result in no or low drug initiation of drug use; and 2) Youth will share more open communication around drug use in focus groups (i.e. higher substance use admittance). Thus, the qualitative data will yield additional information about parent-child communication and substance use. 


\section{Method}

\subsection{Participants}

Quantitative Participants

There were 111 mostly female (59.8\%) participants with a mean of 18.9 years of age ranging from 12 - 47 years old. All participants volunteered to be in the study and were treated in accordance to the American Psychological Association ethical guidelines. The University's Institutional Review Board approved the study protocol. The majority of the participants in this study were African American, comprising $69.1 \%$ of the sample; approximately $4.5 \%$ of the sample identified themselves as Caucasian, .9\% identified themselves as Native American or Indian, and $10 \%$ reported being mixed or biracial. The remainder of the participants checked the "race not listed” option on the survey (15.5\%).

\subsection{Materials}

\subsubsection{Youth Risk Behavior Surveillance System (YRBSS)}

The YRBSS was developed in 1989 by the Centers for Disease Control and Prevention (CDC) to monitor health risk behaviors that contribute to the leading causes of mortality, morbidity, and social problems among youth and adults in the United States. This survey used the sexual behaviors that contribute to unintended pregnancy and sexually transmitted disease category from the scale. The Youth Risk Behavior scale test—retest reliability was reported to be .63 (Brener et al., 2002).

\subsubsection{Family Adaptation and Cohesion Scale (FACES-IV)}

The FACES-IV measures family cohesion, disengagement, and emotional reactivity. This survey used the family cohesion subscale. Participants rated items using a 5 point Likert scale to describe their family interactions. The scale was reported to be valid with a reliability of .87 (Baptist, Thompson, Norton, Hardy, \& Link, 2012; Olson, 2011).

\subsubsection{Parent-Child Relationship Inventory (PCRI)}

The PCRI is intended to measure parental support, satisfaction with parenting, involvement, communication, limit setting, autonomy, and role orientation. The test-retest reliability correlations after one week are as follows: parental support (.81), satisfaction with parenting (.73), involvement (.87), communication (.68), limit setting (.93), autonomy (.78), and role orientation (.89). Chronback's alphas for internal consistency include: parental support (.70), satisfaction with parenting (.85), involvement (.76), communication (.82), limit setting (.88), autonomy (.80), and role orientation (.75).

\subsubsection{Qualitative Participants}

The parents and children were given a "family" identifier so that the researchers could match families for easy statistical analysis and comparability to the qualitative data at a later date. The focus group consisted of 17 participants. The child focus group consisted of eight African American adolescents aged 16 - 17. The parent focus group consisted of nine adults aged $30-47$.

The focus group questions consisted of modified Youth Risk Behavior Surveillance System items to assess their communication with parents about tobacco, marijuana, other illegal drugs (heroin, cocaine, Ecstasy, etc.), and unprescribed use of prescription drugs (Ritalin, Adderall, Valium, Vicodin, Xanax, etc.). For example, the item, “Are you comfortable talking to your parents about your sexual history?” was changed to "Are you comfortable talking to your parents about your drug use?” Similar to the child focus group, items were changed from sexual history to drug use. For example, "Are you comfortable talking to your children about their sexual history?” changed to “Are you comfortable talking to your children about their drug use?” The child and parent focus groups were audio taped and transcribed.

\subsection{Procedures}

Quantitative data was collected during two community events. The data was analyzed using the Statistical Package for the Social Sciences (SPSS). Qualitative data were collected and audio taped during two separate sessions at a local middle school. The child focus groups consisted only of the teen participants and three re- 
search assistants to maintain anonymity of the statements and reduce fear of substance use information getting back to parents. Researchers asked the modified YRBSS questions and participants answered freely. Clarification questions were answered, but no examples of the type of responses expected were given to ensure minimal researcher bias introduction. Parental focus groups were conducted in a similar structure where only the parent participants and researchers were in the room and audio taped. Focus group audiotapes were later transcribed. Thematic analysis as described by Braun \& Clarke (2006) was used to produce our results. The six phases of thematic analysis include familiarizing oneself with the data, generating initial codes, searching for themes, reviewing themes, defining and naming themes, and producing the report. The focus groups were audio taped, transcribed, and checked again for accuracy. Three separate individuals performed coding and analyses and final themes were formed by a fourth individual after viewing the three individual themes.

\section{Results}

\subsection{Quantitative Results}

Approximately $27.9 \%(n=31)$ of the sample admitted to at least trying tobacco, $65.8 \%(n=73)$ admitted to having at least one drink of alcohol, 40.5\% ( $\mathrm{n}=45)$ admitted to using marijuana, 2.7\% $(\mathrm{n}=3)$ admitted to using some form of cocaine, and $10.8 \%(\mathrm{n}=12)$ admitted to taking prescription drugs without a doctor's prescription. Overall, 67.6\% $(n=75)$ of the participants stated that they had used any one of these substances at one time during their lives. Approximately $19.8 \%$ of the sample reported they had never tried alcohol, $50.5 \%$ admitted they took their first drink of alcohol around the age of 13 or older, $8.1 \%$ took their first drink between the ages of 10 - 11, and 5.4\% admit that they were between 8 - 9 years old when they took their first drink of alcohol. Approximately $41.1 \%$ admitted they had never tried marijuana, 39.6\% admitted they tried marijuana at age 13 or older, and $2.7 \%$ admitted they tried marijuana at age 10 or older. The data was separated to allow a comparison of the youth and parent information. Of those participants 17 and under: 22 out of the 31 (70.97\%) participants had tried a substance (not including tobacco products) and 23 out of the 31 (74.19\%) of the participants had tried a substance (alcohol, drugs, tobacco). Of the participants 18 and older: 44 out of the 62 (70.97\%) participants had tried drugs, marijuana, or alcohol by age 12 or before (mostly marijuana).

Pearson correlations were performed to assess whether substance use was associated with family cohesion, family communication, and parental relationships. Family cohesion, family communication, and parental relationships were obtained by summing items on a scale of the YRBSS. The Pearson correlations are reported in Table 1. The results are indicated below.

\subsubsection{Family Cohesion}

It was hypothesized that the greater the amount of family cohesion, the less likely the child would be to engage in substance use. A statistically significant Pearson correlation, $r(89)=-.281, p<.001$ (two-tailed) indicated that the more cohesive a family is, the less likely the participant would be to engage in substance use.

\subsubsection{Family Communication}

It was hypothesized that the greater the amount of family communication, the less likely the child would be to

Table 1. Correlations between family cohesion, family communication, parental relationships, first sexual intercourse, and substance use ( $\mathrm{N}=91$ participants).

\begin{tabular}{cc}
\hline & Substance Use \\
\hline Family Cohesion & $-.281^{* *}$ \\
Family Communication & $-.298^{* *}$ \\
Parental Relationship & -.055 \\
First Sexual Intercourse & $.248^{*}$ \\
\hline
\end{tabular}

Note: Bonferroni corrected Pearson correlation alpha levels were used to control for the inflated risk of Type I error, which occurs when multiple significance tests are performed. Using this more conservative criterion for statistical significance, family cohesion and family communication were still significant. ${ }^{* *} p<.01$, two-tailed. ${ }^{*} p<.05$, two-tailed. 
participate in substance use. A statistically significant Pearson correlation, $r$ (88) $=-.298, p<.001$ (two-tailed) indicated that the stronger the family communication, the less likely participants were to engage in substance use.

\subsubsection{Parental Relationship}

It was hypothesized that the stronger the parental relationship, the less likely the child would be to engage in substance use. However, parental relationship and substance use were not statistically significant.

\subsubsection{Substance Use}

A Pearson correlation was performed to assess whether substance use was associated with the age of first sexual intercourse. The correlation between substance use and the age of first sexual intercourse was statistically significant, $r(89)=+.248, p<.005$ (two-tailed). As indicated in prior research studies (Muchimba, Haberstick, Corley, \& McQueen, 2013), the individuals who used drugs were more likely to have an earlier onset of first sexual intercourse and less condom use than their counterparts who did not use drugs in our study. The individuals who used drugs were also more likely to have multiple partners. In addition, amongst the individuals that used drugs, the higher the substance use levels, the younger the age of first sexual intercourse.

\subsection{Qualitative Results}

Thematic analysis, as described by Braun \& Clarke (2006), was used to produce our results. The focus groups were audio taped, transcribed, and checked again for accuracy. Three separate individuals performed coding and analyses and final themes were formed and defined by a fourth individual after viewing the three individual themes (see Table 2 for qualitative data extraction example). Based on this process, the consistent themes are below:

Theme 1: The youth participants felt comfortable talking to their parents about drugs as long as it wasn't discussing their personal use. This theme appears to fit with the quantitative data that was collected.

Theme 2: Most of the information that the youth participants received about drugs they had received from other sources than their parents. In addition, most of the information the parent participants had received about drugs had come from other sources. $100 \%$ of the parent focus group participants had no recollections of ever talking to their parents about drugs.

Theme 3: The information about drugs youth participants received from their parents was about not doing those drugs, not actual information about the symptoms or harmful effects of the drugs.

Theme 4: Most of the participants did not tell their parents about their use of drugs. Parents believed their children had most likely viewed others using drugs, but they (their children) would not use drugs.

It was hypothesized that there would be greater admittance to drug use in the focus groups than on the quantitative measurements. This appeared to be the case, with seven out of eight participants, or $87.5 \%$, admitting to some type of substance use compared to the quantitative sample with $67.6 \%$ of the participants admitting to some type of substance use.

\section{Discussion}

The first major finding showed that our data on a primarily African American population mirrored or was very similar to the results on primarily Caucasian samples with regard to communication between parents and children and its overall protective effect against substance abuse. While our effect size appears small, it is in the hypothesized direction and is statistically significant. These data on African Americans is of particular interest given the rates of substance abuse in the US in general and the African American community specifically. The association of parental communication and drug use indicated a need to further explore the dynamics of the sub-

\section{Table 2. Qualitative data extraction example.}


stance abuse information and communication between parents and youth. While $70.97 \%$ of the youth in this study admitted to using drugs, "all" of the parents of these youth said they were certain their child did not use drugs. In addition, the youth suggested that their parents state, "Do not use drugs". However, they do not engage in deeper conversations such as the consequences of using drugs, parental disappointment surrounding drug use, the visualization of drug use in the community, and other relevant factors that may influence drug use among youth. This finding is consistent with Miller-Day \& Kam (2010), who discuss the need to identify targeted messages related to parent-child communication and alcohol use.

The results from this study suggest that communication and family cohesion both relate to drug use. This finding alone demonstrates the need for research on more specific ways in which family cohesion may prevent drug use and more specifically, the type of cohesion that produces a protective factor to buffer risk of drug use/abuse in children and adolescents. Other research that could add substantively to this field includes research on the types of family communication processes that offer protection against drug use. For those professionals working within a Multisystemic Therapy model and specifically those performing intensive in-home therapy and intensive outpatient therapy, these findings are of particular importance. First, these findings support a family cohesion approach to working with youth. Families that demonstrate greater cohesiveness demonstrated less substance abuse than those who had less cohesion. Those practitioners who are working with youth who have substance abuse problems and who are seeking to increase family cohesion may be on the right track. What is not certain from this research is the type of cohesion or if there are moderator variables. For instance, families may have high levels of cohesion, but be committed to antisocial practices, and thus, substance abuse may present itself among the youth of that family. This stands in contrast to those who express family cohesion, but are more pro-social in their familial world view.

There were definitely limitations to our study such as the sample size, the results are limited to the African American population, and survey data may yield some socially desirable answer selections. However, our findings demonstrate that the types of communication offered by parents may be of critical importance in assisting children and youth in avoiding drug use. Again, the issue of how these communications produce this buffering effect is of interest and should be considered for future studies. Studies that identify specific types of communication and even types of cohesiveness and then submit that data to structural models may offer insights and some specificity as to how these constructs offer a protective factor. Furthermore, future studies may also explore the possible effects of specific parental communication styles and authorship of the communication (father versus mother) on substance abuse.

\section{References}

American Psychiatric Association (2000). Diagnostic and Statistical Manual of Mental Disorders (4th ed.). Washington DC: American Psychiatric Association.

Baptist, J. A., Thompson, D. E., Norton, A. M., Hardy, N. R., \& Link, C. D. (2012). The Effects of the Intergenerational Transmission of Family Emotional Processes on Conflict Styles: The Moderating Role of Attachment. American Journal of Family Therapy, 40, 56-73. http://dx.doi.org/10.1080/01926187.2011.575030

Bauermeister, J. A., Zimmerman, M. A., Johns, M. M., Glowacki, P., Stoddard, S., \& Volz, E. (2012). Innovative Recruitment Using Online Networks: Lessons Learned from an Online Study of Alcohol and Other Drug Use Utilizing a Web-Based, Respondent-Driven Sampling (webRDS) Strategy. Journal of Studies on Alcohol and Drugs, 73, 834-838.

Braun, V. \& Clarke, V. (2006) Using Thematic Analysis in Psychology. Qualitative Research in Psychology, 3, 77-101. http://dx.doi.org/10.1191/1478088706qp063oa

Brener, N. D., Kann, L., McManus, T., Kinchen, S. A., Sundberg, E. C., \& Ross, J. G. (2002). Reliability of the 1999 Youth Risk Behavior Survey Questionnaire. Journal of Adolescent Health, 31, 336-342. http://dx.doi.org/10.1016/S1054-139X(02)00339-7

Dawson, D. A., Grant, B. F., Stinson, F. S., \& Chou, P. S. (2004). Another Look at Heavy Episodic Drinking and Alcohol Use Disorders among College and Noncollege Youth. Journal of Studies on Alcohol, 65, 477-488.

Demant, J., \& Ravn, S. (2013). Communicating Trust between Parents and Their Children: A Case Study of Adolescents' Alcohol Use in Denmark. Journal of Adolescent Research, 28, 325-347. http://dx.doi.org/10.1177/0743558413477198

Elkington, K. S., Bauermeister, J. A., \& Zimmerman, M. A. (2011). Do Parents and Peers Matter? A Prospective SocioEcological Examination of Substance Use and Sexual Risk among African American Youth. Journal of Adolescence, 34, 1035-1047. http://dx.doi.org/10.1016/j.adolescence.2010.11.004

Haas, A. L., Smith, S. K., Kagan, K., \& Jacob, T. (2012). Pre-College Pregaming: Practices, Risk Factors, and Relationship 
to Other Indices of Problematic Drinking during the Transition from High School to College. Psychology of Addictive Behaviors, 26, 931-938. http://dx.doi.org/10.1037/a0029765

Luk, J. W., Farhat, T., Iannotti, R. J., \& Simons-Morton, B. G. (2010). Parent-Child Communication and Substance Use among Adolescents: Do Father and Mother Communication Play a Different Role for Sons and Daughters? Addictive Behaviors, 35, 426-431. http://dx.doi.org/10.1016/j.addbeh.2009.12.009

Miller-Day, M., \& Kam, J. A. (2010). More than Just Openness: Developing and Validating a Measure of Targeted ParentChild Communication about Alcohol. Health Communication, 25, 293-302. http://dx.doi.org/10.1080/10410231003698952

Muchimba, M., Haberstick, B. C., Corley, R. P., \& McQueen, M. B. (2013). Frequency of Alcohol Use in Adolescence as a Marker for Subsequent Sexual Risk Behavior in Adulthood. Journal of Adolescent Health, 53, 215-221. http://dx.doi.org/10.1016/j.jadohealth.2013.02.005

Olson, D. (2011). FACES IV and the Circumplex Model: Validation Study. Journal of Marital and Family Therapy, 37, 64-80. http://dx.doi.org/10.1111/j.1752-0606.2009.00175.x

Pacek, L. R., Malcolm, R. J., \& Martins, S. S. (2012). Race/Ethnicity Differences between Alcohol, Marijuana, and Co-Occurring Alcohol and Marijuana Use Disorders and Their Association with Public Health and Social Problems Using a National Sample. The American Journal on Addictions, 21, 435-444. http://dx.doi.org/10.1111/j.1521-0391.2012.00249.x

Stanton, B., Cole, M., Galbraith, J., Li, X., Pendleton, S., Cottrel, L., Marshall, S., Wu, Y., \& Kaljee, L. (2004). Randomized trial of a Parent Intervention: Parents Can Make a Difference in Long-Term Adolescent Risk Behaviors, Perceptions, and Knowledge. Archives of Pediatrics and Adolescent Medicine, 158, 947-955. http://dx.doi.org/10.1001/archpedi.158.10.947

Substance Abuse and Mental Health Services Administration (2003). Overview of Findings from the 2002 National Survey on Drug Use and Health. Rockville, MD: Substance Abuse and Mental Health Services Administration.

Substance Abuse and Mental Health Services Administration (2012). Results from the 2011 National Survey on Drug Use and Health: Mental Health Findings. Rockville, MD: Substance Abuse and Mental Health Services Administration.

Turrisi, R., Mastroleo, N. R., Mallett, K. A., Larimer, M. E., \& Kilmer, J. R. (2007). Examination of the Mediational Influences of Peer Norms, Environmental Influences, and Parent Communications on Heavy Drinking in Athletes and Nonathletes. Psychology of Addictive Behaviors, 21, 453-461. http://dx.doi.org/10.1037/0893-164X.21.4.453

Whitaker, D. J., \& Miller, K. S. (2000). Parent-Adolescent Discussions about Sex and Condoms: Impact on Peer Influences of Sexual Risk Behavior. Journal of Adolescent Research, 15, 251-273. http://dx.doi.org/10.1177/0743558400152004

Wills, T. A., Gibbons, F. X., Gerrard, M., Murry, V. M., \& Brody, G. H. (2003). Family Communication and Religiosity Related to Substance Use and Sexual Behavior in Early Adolescence: A Test for Pathways through Self-Control and Prototype Perceptions. Psychology of Addictive Behaviors, 17, 312-323. http://dx.doi.org/10.1037/0893-164X.17.4.312 
Scientific Research Publishing (SCIRP) is one of the largest Open Access journal publishers. It is currently publishing more than 200 open access, online, peer-reviewed journals covering a wide range of academic disciplines. SCIRP serves the worldwide academic communities and contributes to the progress and application of science with its publication.

Other selected journals from SCIRP are listed as below. Submit your manuscript to us via either submit@scirp.org or Online Submission Portal.
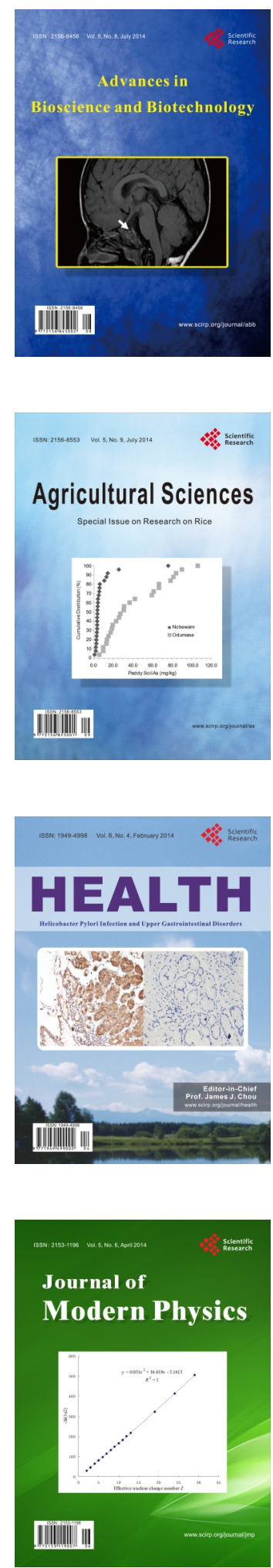
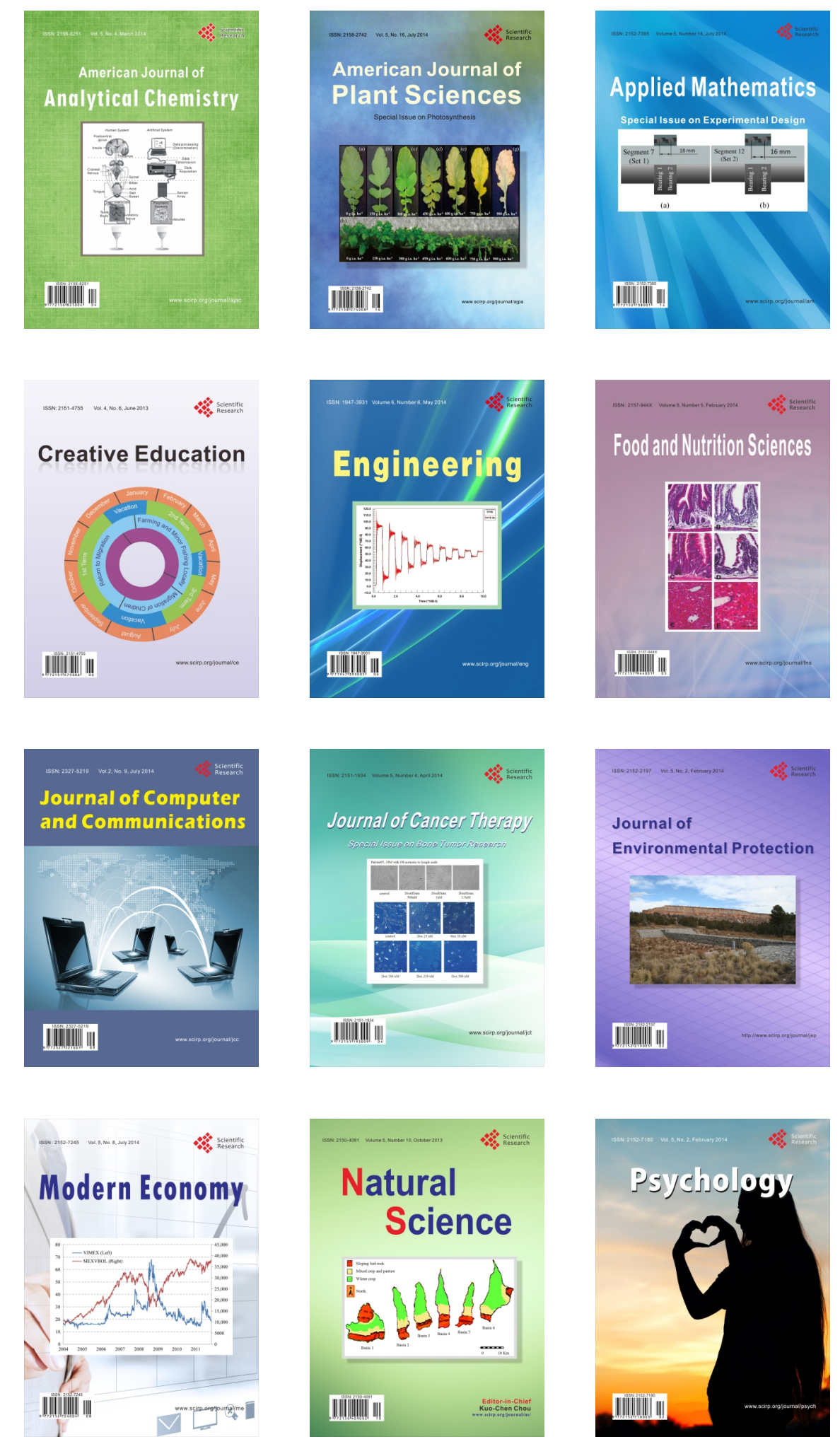